standard operating procedures, and recommendations to overcome the common challenges within conflict contexts.

Conclusion: The outcome document will be used as a reference guide to build on existing knowledge in mobile health service delivery in complex emergencies.

Prehosp Disaster Med 2017;32(Suppl. 1):s86-s87

doi:10.1017/S1049023X17002266

Prevention and Risk Management from Nature to Society: How can Medicine Help Reduce the Refugee Crisis in Mexico?

Joanne Joloy

Ya Respondiste A.C, Ciudad de México/Mexico

Study/Objective: To sensitize Mexican doctors on preventing immigrant disease complications.

Background: Mexico serves as a halfway point for immigrants seeking safety and a better life. Many of these refugees carry severe diseases that complicate matters. These complications could be prevented by proper and timely treatment. Currently, there are no programs targeted at immigrant health. Doctors should be sensitized to the current situation and the need to prevent health complications.

Methods: Retrospective, observational, longitudinal study of refugee patients found in Mexico City; 200 refugee patients (ages 11 to 55) found in immigrant homes and hotels working with the Responde A.C. Foundation were included.

1. Three different sites were selected, where there was a higher density of patients.

2. Specialized and experienced doctors enrolled the patients through a weekly triage.

3. A clinical file was created for each patient containing a full medical history and background on the patient. 4. Patients were given a weekly follow-up for 2 months, during these follow-ups they were asked about their recovery progress. A clinical consult was given to monitor their progress.

Results: We found that there is a constant need for general and specialized medical care among refugees in Mexico. Providing timely and appropriate care showed improvement in the patients perceived quality of life. The 130 patients showed good progress, 20 patients developed complications, and 50 patients were lost during follow-up. At the present time, the statistical analysis will conclude by the end of 2016 .

Conclusion: There is an immigrant crisis in Mexico, and health-care providers should be aware of this problem and action should be taken to prevent this crisis from continuing to grow. This study shows the importance of timely and accurate medical care, and the impact it could have in preventing complications and improving the quality of life of refugees in Mexico.

Prehosp Disaster Med 2017;32(Suppl. 1):s87

doi:10.1017/S1049023X17002278

The Educational Challenge in 2017: Providing Emergency Care to Migrants and Vulnerable People

Alessandro Barelli, Camilla Naso

European Center for Disaster Medicine, San Marino/San Marino
Study/Objective: The aim of the study was to explore and describe problems and difficulties in situations with migrants in emergency care in order to plan for an appropriate and effective teaching program for emergency care providers.

Background: In 2016, there were many posed threats to emergency medicine practitioners all over Europe. Terrorism, climate changes, and seasonal diseases could be sufficient to challenge emergency medical systems; they add to the hardships facing the thousands of migrants who continue to cross into Europe with the never-ending conflict in Syria and Iraq. In those settings, emergency medicine must play its key role of being the last resort for people with no primary health care and no alternative.

Methods: An explorative study was carried out using a sample of 67 emergency care providers, 15 physicians, 52 registered nurses, 46 women, and 21 men experienced in emergency care. They documented their experiences of problematic situations.

Results: The health care professionals' experiences of problematic situations with migrants in emergency care were described as: (1) language barriers; (2) reliance on authorities; (3) different behavior; (4) contact with relatives; (5) complicating logistic factors; (6) gender roles; (7) patient's earlier experiences of violence; (8) use of natural remedies; and (9) lack of knowledge on specific health care problems of migrants.

Conclusion: Results showed the main problem was related to communication difficulties, including language barriers and cultural dissimilarities. Another key factor is the lack of knowledge on specific health care problems of migrants. In order to mitigate the problems, the use of adequate interpreters is a theoretical chance, whereas using language-free communication tools (cartoons and vignettes) could be a financially effective alternative. Training programs for emergency care providers must include sessions to improve knowledge about the care of migrants from different parts of the world. The importance of searching for the unique individual perspective is stressed.

Prehosp Disaster Med 2017;32(Suppl. 1):s87

doi:10.1017/S1049023X1700228X

Evidence: Aid Special Collection for the Health of Refugees and Asylum Seekers

Claire Allen ${ }^{1}$, Jeroen Jansen ${ }^{2}$, Tony Aburrow ${ }^{3}$

1. Evidence Aid, Evidence Aid, Oxford/United Kingdom

2. Evidence Aid, Oxford/United Kingdom

3. John Wiley and Sons Limited, Chichester/United Kingdom

Study/Objective: To build collections of health care evidence, to provide those addressing the health of refugees with some guidance, collections divided between Evidence Aid and Cochrane.

Background: In 2015, over one million people arrived in Europe by sea, mostly originating from Syria. In the same year, 3,771 people went missing or died attempting to reach safety in Europe. In 2016, people continue to make the hazardous journey across the sea, and at the beginning of February, 67,072 people made it across, while 357 were reported dead or missing. Methods: Both collections focus on some of the most relevant medical conditions, as perceived by experts involved, either in 
guideline development or on the frontline, directly addressing the health care needs of refugees and asylum seekers. In the first instance, the work-group addressed five priority conditions.

Results: The collection "Health of Refugees and Asylum Seekers in Europe" was published on December 2, 2016, hosting curated resources from the Cochrane Library and other research outputs, categorized into guidelines; systematic reviews; articles; and other information.

Conclusion: Since publication, the refugee health collection, found on the website EvidenceAid.org, has received almost 600 page views, ranking it third amongst most viewed pages after the homepage and the resources tab for that period. On average, users have been spending 2.30 minutes on the page, suggesting the content is commanding attention. We will continue to encourage an evidence-based response to this crisis, and will report on usage of both collections at the conference.

Prehosp Disaster Med 2017;32(Suppl. 1):s87-s88

doi:10.1017/S1049023X17002291

\section{Ethics and Palliative Care During International}

\section{Humanitarian Action}

Mollie Sivaram ${ }^{1}$, Elysee Nouvet ${ }^{2}$, Matthew Hunt ${ }^{3}$, Lisa Schwartz'

1. Health Sciences, McMaster University, Hamilton/ON/Canada

2. Clinical Epidemiology And Biostatistics, McMaster University, Hamilton/Canada

3. School Of Physical And Occupational Therapy, McGill University, Montreal/QC/Canada

Study/Objective: This is a critical interpretive synthesis of the ethical and practical limitations inherent to the provision of palliative care by humanitarian organizations during public health emergencies. Developed in dialogue with the SPHERE Project, the Palliative Care in Complex Humanitarian Emergencies network, and the Children's Palliative Care Network, and was situated within a broader qualitative study on the place of and need for palliative care in humanitarian crises. Its findings can be adapted to inform guidelines for aid agencies for the provision of palliative care.

Background: Standards of care in crisis settings emphasize that patients who are dying should be treated with respect and properly attended to. However, in humanitarian crisis where demands for care outweigh resources, efforts are typically directed to those most likely to survive. The need for humanitarian agencies to provide palliative care was recently highlighted in the Ebola crisis, it is still unclear if, and how 'end-of-life' care is approached in context. Aid workers need to be equipped with the ability to initiate palliative treatment as a worthy intervention, even in times of crisis and scarcity.

Methods: Literature was captured using standardized, key and mesh term searches through academic databases, including MEDLINE, EMBASE, CINAHL, Web of Science, as well as grey literature databases (ReliefWeb, IGO).

Results: Findings described here include: (1) the interaction of humanitarian principles with triage priorities in disasters; (2) the politics and ethics of healthcare exclusion; (3) 3-case studies exemplifying the need for increased guidance and preparation for palliative care needs in humanitarian emergencies.
Conclusion: This Research for Health in Humanitarian Crises (R2HC)-funded analysis will help elucidate the realities of palliative care needs in humanitarian practice, and inform the development of guidelines and training to better prepare humanitarian healthcare teams for palliative and end-of-life needs in the field.

Prehosp Disaster Med 2017;32(Suppl. 1):s88

doi:10.1017/S1049023X17002308

Epidemiological Humanitarian Aid: Data for Evidenced Based Decision Making in Disaster and Conflict Medicine John M. Quinn $V^{1}$, Vitaliy Krylyuk ${ }^{2}$, Olia Romaniuk ${ }^{3}$, Patrick Chellew ${ }^{4}$, Vladimir Bencko ${ }^{5}$

1. Expert Consultant, Reforms Office (ro) At The Ministry Of Defense (mod) In Ukraine, PhD Candidate and Researcher, Prague Center for Global Health, Institute of Hygiene and Epidemiology, First Faculty of Medicine, Charles University, Praha/Czech Republic

2. Emergency Medicine, Ukrainian Resuscitation Council, Kyiv/ Ukraine

3. Program Researcher, Medical ATO Report Series, Kyiv/Ukraine

4. Program Lead, Medical ATO Report Series, Kyiv/Ukraine

5. Prague Center for Global Health, Institute of Hygiene and Epidemiology, First Faculty of Medicine, Charles University in Prague, Praha/Czech Republic

Study/Objective: Qualitative and quantitative assessment of disaster and Ukraine ATO/Contact Line to report the incidence and prevalence of communicable and noncommunicable disease (NCD) (to include trauma) to better serve policy and decision makers on humanitarian aid packages. A thorough assessment tool for civil and military medical needs, gaps, and reporting that feed into all disaster services to enable evidencedbased decision making.

Background: Currently, many barriers and challenges remain for donors, humanitarian actors, and governmental institutions to appropriately allocate resources due to the lack of sound data and epidemiological principles. Systems and standards for disaster preparedness, prehospital medicine, evacuation chain management, and noncommunicable disease are lacking and require evidenced-based decision making at a policy level.

Methods: A thorough, quantitative, and qualitative descriptive analysis and updated stakeholder mapping to describe the methods for carrying out emergency operations, the process for rendering mutual aid, the emergency services of governmental agencies requiring interoperability, analyze how resources are mobilized, how the public and other agencies will be informed, and the process to ensure continuity of government and core functions, such as rule of law, during an emergency or disaster and all other medical services. This must include a data-driven epidemiological core focus based on data, applicable to any manmade or natural disaster, and be sustainable in nature. This must be testable, reproducible, and well-versed across agencies. Medical support elements and security assets may create a clear picture that will enhance support for both Ukraine $\mathrm{MoD}$, Ukraine $\mathrm{MoH}$, and other NATO-associated partners.

Results: Assessment Reports and data tools able to sustainably highlight disaster preparedness, evacuation chain management, 